\title{
ACEPTABILIDAD Y CONSUMO DE UNA BEBIDA LÁCTEA CON OMEGA-3 EN EMBARAZADAS Y NODRIZAS DEL PROGRAMA NACIONAL DE ALIMENTACIÓN COMPLEMENTARIA
}

\section{ACCEPTABILITY AND CONSUMPTION OF A DAIRY DRINK WITH OMEGA-3 IN PREGNANT AND LACTATING WOMEN OF THE NATIONAL SUPPLEMENTARY FOOD PROGRAM}

\author{
Angela Contreras M. (1), Yilda Herrera (1), Lorena Rodríguez O. (1), \\ Tito Pizarro Q. (1), Eduardo Atalah S. (1,2)
}

(1) Departamento de Alimentos y Nutrición, División de Políticas Públicas Saludables y Promoción, Subsecretaria de Salud Pública, Ministerio de Salud, Santiago, Chile.

(2) Departamento de Nutrición, Facultad de Medicina,

Universidad de Chile, Santiago, Chile.

\begin{abstract}
Background: The Ministry of Health incorporated in 2008 in the national supplementary feeding program a milk based drink for pregnant and nursing women fortified with EPA and DHA. The product initially presented some degree of rejection in the population, which led to modify its organoleptic characteristics. Objective: To evaluate in a national sample the acceptability and consumption of the modified product a year after starting distribution. Methodology: Random sample of 1,272 beneficiaries in 62 clinics and 21 rural health posts across the country. $A$ semi-structured survey to assess withdrawal, acceptability and consumption by the beneficiary and other household members was applied. Results: Ninety point seven \% of mothers regularly withdrew the product. There were important problems in the preparation of "Purita Mama" by lower dilution (22\%) or excessive concentration (50.8\%). The overall assessment of the product showed an average of 3 (regular) on a scale of 1 to 5 in pregnant and lactating women, highlighting negative opinions about the excessive sweetness (19.2\%) and strong aroma (49.9\%). The median overall assessment of the mothers was 3 (regular) on a scale of 1 to 5, without significant differences between pregnant and lactating women. The median consumption of the beneficiaries was $250 \mathrm{ml} /$ day, close to schedule and higher in the nursing women, although they receive more product. Eighty $\%$ of mothers appreciate the nutritional benefits of the product to the mother or child. About $60 \%$ of mothers do not share the milk drink, which indicates a relatively low domestic dilution. Conclusions: there was no significant change in the level of acceptability of the product and the consumption levels were relatively adequate. It is important to enhance information on how to prepare the product, to propose different alternatives for consumption and eventually to consider alternatives of flavor. Key words: pregnant; nursing woman; food programs; milk based drink; evaluation.
\end{abstract}

Este trabajo fue recibido el 27 de Abril de 2011 y aceptado para ser publicado el 2 de Junio de 2011.

\section{INTRODUCCIÓN}

Los ácidos grasos poliinsaturados de cadena larga omega-3 de origen marino son esenciales a través de todo el ciclo vital, especialmente el ácido eicosapentaenoico (EPA) y docosahexaenoico (DHA). Un mayor consumo de estos ácidos grasos en el embarazo y lactancia se ha asociado a un mejor desarrollo cognitivo y visual del niño los primeros años de vida y a menor riesgo de prematuridad (1-4). También se han demostrado efectos positivos en la salud cardiovascular (5-7) y mental del adulto y adulto mayor (8-11).

El ácido alfa linolénico, precursor del EPA y DHA, que está presente en aceites vegetales, no logra los mismos efectos biológicos, por el bajo grado de transforma- 
ción en el organismo hacia ácidos grasos poliinsaturados de cadenas mas largas (12-14).

No hay consenso sobre los niveles de consumo óptimo de DHA en las distintas etapas de la vida, pero la mayor parte de los grupos técnicos lo sitúan entre 200 y $500 \mathrm{mg} /$ día en la población adulta $(2,14,15)$. En Chile, el consumo de pescado o de otros alimentos de origen marino es muy bajo, lo que determina un aporte estimado de DHA del orden de sólo $50 \mathrm{mg}$ /día (16), muy por debajo de las recomendaciones y de lo observado en otros países $(4,12,17)$.

En agosto del 2008 el Ministerio de Salud incorporó al Programa Nacional de Alimentación Complementaria (PNAC) una bebida láctea con ácidos grasos omega-3, en reemplazo de la leche entera fortificada con hierro, zinc, cobre y vitamina $\mathrm{C}$ que se distribuía los últimos 10 años. El nuevo producto tiene $10 \%$ de materia grasa, es reducida en lactosa y está fortificado con 10 vitaminas, 4 minerales y aporta $79 \mathrm{mg}$ de EPA + DHA por porción de consumo habitual del producto $(200 \mathrm{~mL})$. Un estudio piloto demostró buena aceptabilidad del producto y un aumento importante en el consumo de DHA de la madre y en la concentración de DHA en la membrana del glóbulo rojo de la embarazada y en la leche materna $(18,19)$.

A cinco meses de haberse iniciado la distribución de la bebida láctea Purita Mamá, el Ministerio de Salud realizó una encuesta a nivel nacional para evaluar la opinión de las madres respecto al nuevo producto. El estudio demostró una moderada aceptabilidad por parte de las usuarias, principalmente por un acentuado olor y sabor a vainilla (20). Ello determinó la necesidad de desarrollar nuevos prototipos que fueron evaluados por paneles de expertos y posteriormente en un estudio piloto en dos centros de atención primaria de la Región Metropolitana y del Bío-Bío. En base a estos resultados, se empezó a distribuir a partir de Octubre de 2009, la "Purita Mamá modificada" con reducción del dulzor y del sabor a vainilla en relación al producto original.

El objetivo del estudio es evaluar por medio de una encuesta a nivel nacional, la percepción de las madres beneficiarias del programa sobre las características organolépticas y el nivel de consumo de este nuevo producto.

\section{SUJETOS Y MÉTODOS}

Se seleccionó una muestra aleatoria, estratificada, por etapas, representativa de las embarazadas y nodrizas controladas en todas las SEREMI del país. Para definir el tamaño muestral se estimó un 50\% de aceptabilidad del producto, un $10 \%$ de error en la estimación, un nivel de significación $<0,01$ y un poder del test de 90 , lo que determinó la necesidad de estudiar 368 embarazadas e igual número de nodrizas. Se optó por estudiar una mues- tra 2,2 veces mayor para poder analizar la información en relación a otras variables de de interés: trimestre del embarazo, meses de lactancia y hábito de consumo de leche previo al embarazo, entre otras.

El programa distribuye $1 \mathrm{~kg}$ de bebida láctea al mes en embarazadas de peso normal, sobrepeso u obesidad, lo que permite preparar cerca de $260 \mathrm{~mL} /$ día, diluida al 12,5\% según la recomendación del Ministerio de Salud. En el caso de las nodrizas, los $2 \mathrm{~kg}$ que reciben la mayoría de ellas, les permitiría, consumir cerca de $500 \mathrm{~mL} /$ día.

Se diseñó una encuesta con 17 preguntas semi estructuradas, orientadas a conocer el nivel de consumo previo de leche, el consumo de Purita Mama y la evaluación de la beneficiaria en cuanto a olor, textura y sabor del nuevo producto. También se exploró si las madres habían recibido información sobre las características nutricionales del alimento y el eventual consumo por otros miembros de la familia. Las preguntas fueron redactadas con un lenguaje simple y comprensible para la población en estudio. La apreciación global de las beneficiarias sobre el producto fue medida en una escala de 1 a 5, que consideraba las categorías muy mala, mala, regular, buena, muy buena.

La encuesta fue aplicada por profesionales del sistema de salud en 62 consultorios urbanos o centros de salud familiar y en 21 postas rurales de las 15 Regiones de país. La muestra fue seleccionada entre las madres que esperaban control de salud o que estaban retirando alimentos del PNAC, previo consentimiento informado. El trabajo de terreno fue coordinado por los encargados de nutrición y programas alimentarios de cada SEREMI. Las encuestas fueron revisadas a nivel de cada SEREMI y posteriormente enviadas al Departamento de Alimentos y Nutrición del Ministerio de Salud, donde fueron digitadas y procesadas. Se utilizó un procedimiento de control para verificar la calidad de la digitación y para revisar las encuestas con respuestas inconsistentes.

Se analizó la normalidad de las variables contínuas y dado que, la mayoría de ellas no tenía una distribución normal, se utilizaron medianas y percentiles como variables de tendencia central y el test de Kruskall Wallis para la comparación entre grupos. Las variables categorías se analizaron por distribución de frecuencia y se compararon con la prueba de chi2, considerando como significativo un $\mathrm{p}<0,05$.

\section{RESULTADOS}

Se encuestaron 1.272 beneficiarias, con una proporción muy similar de embarazadas y nodrizas $(49,8$ y 50,2\% respectivamente), siendo la mayor parte de ellas residentes en áreas urbanas $(89,5 \%)$ y el resto en localidades rurales. 
El $90,7 \%$ de las madres retiraba regularmente el producto, lo que refleja una buena adherencia al programa, con un comportamiento similar en embarazadas y nodrizas. La principal causa de no retiro del producto fue una baja aceptabilidad (6,0\% del total de la muestra) y en menor proporción por intolerancia, inasistencia a control, indicación médica u otras causas (datos no mostrados).

La encuesta mostró importantes problemas en la forma de preparar la bebida láctea Purita Mamá, que según la norma del programa, debiera diluirse al $12,5 \%$. El 50,8\% de las madres la preparaba muy concentrada (> $15 \%$ ) y un $22,2 \%$ muy diluida $(<10 \%)$, sin diferencias entre embarazadas y nodrizas, lo que sin duda puede afectar la apreciación organoléptica del producto (datos no mostrados).

La opinión de las madres sobre las diferentes características organolépticas de Purita Mamá se muestra en la tabla 1. La apreciación sobre el dulzor muestra una mejoría importante en respecto a la encuesta del
2009, aunque aún una de cada 5 madres considera que el producto es muy dulce. En cuanto a la textura existe bastante heterogeneidad de las respuestas, lo que en parte podría ser explicado por la inadecuada preparación del producto en muchas beneficiarias. Cerca de la mitad de las madres aún considera "fuerte" el olor o aroma de Purita Mamá, lo que sugiere la necesidad de reducir aún más el nivel de saborizante que se usa actualmente. Sólo el 18\% de las madres consideró que el sabor de Purita Mamá era característico de la leche. Todas estas apreciaciones tuvieron un comportamiento similar en embarazadas y nodrizas.

La apreciación global de las madres sobre el producto en una escala de 1 a 5 tuvo una mediana de 3 (regular), con percentiles 25 y 75 de 2 y 4 , sin diferencias significativas entre embarazadas y nodrizas (datos no mostrados). Aproximadamente un tercio de las madres consideró el producto menos que regular, un tercio regular y el resto bueno, reflejando por lo tanto una gran heterogeneidad

\section{TABLA 1}

\section{Evaluación de las características organolépticas de la bebida láctea Purita Mamá, según tipo de beneficiaria.}

\section{Característica organoléptica}

\section{Dulzor}

Poco dulce

Medianamente dulce

Muy dulce

Total

$\chi^{2} \mathrm{p}$

Textura

Muy aguada

Adecuada

Muy espesa

Total

$\chi^{2} \mathrm{p}$

\section{Olor o aroma}

Suave

Medianamente fuerte

Fuerte

Total

$\chi^{2} \mathrm{p}$

Sabor

Característico de la leche

Sabor extraño

Total

$\chi^{2} \mathrm{p}$

\section{Embarazadas}

$\%$

35,6

45,9

18,5

100,0

$\%$

43,2

44,1

12,7

100,0

$\%$

17,7

31,2

51,1

100,0

$\%$

17,3

82,7

100,0

(5

(

8

(2)

.

$$
6,67<0,05
$$

,

10

$0,92 \mathrm{NS}$

$\begin{array}{cc}19,0 & 18,3 \\ 32,2 & 31,8 \\ 48,8 & 49,9 \\ 100,0 & 100,0\end{array}$

\section{$0,70 \mathrm{NS}$}

46,7

12,7

100,0

Total

$\%$

32,0

48,8

51,7

19,2

100,0

100,0

$\%$
40,6
46,7
12,7
100,0

$\%$

41,9

45,4

12,7

100,0

$\%$

18,3

31,8

49,9

100,0

$\%$

17,8

18,3

82,2

81,7

100,0

\section{0,19 NS}


en la apreciación. Esta situación es similar a la observada en la encuesta previa, lo que hace difícil identificar un alimento de gusto más "universal".

Antes del embarazo un tercio de las madres no tomaba leche y un $25 \%$ adicional consumía una taza o menos al día, lo que sin duda limita la posibilidad de un consumo adecuado durante la gestación. En las beneficiarias del programa el consumo aumentó significativamente con relación a lo observado en la etapa previa al embarazo, disminuyendo casi a la mitad (de $35,1 \%$ a $17,3 \%$ ) las mujeres que no consumían leche y aumentando la proporción $(13,1 \%$ a 22,4\%) de mujeres con una ingesta superior a dos tazas diarias (tabla 2). La mediana de consumo de las embarazadas fue $250 \mathrm{~mL} /$ día, cifra cercana a lo programado. El nivel de consumo fue algo superior en las nodrizas que en las embaraza- das, aunque es importante recordar que la mayoría de ellas recibe el doble del producto, dependiendo del tipo de lactancia (exclusiva, parcial, destete) y del estado nutricional de la madre.

Un alto porcentaje de las madres valora las características del producto, por sus ventajas nutricionales para ella o para su hijo (tabla 3). Ello refleja que han recibido información del equipo de salud, sobre las cualidades del producto, aunque es importante reforzar este mensaje considerando que el $13 \%$ de ellas desconoce por qué se entrega la bebida láctea Purita Mamá o lo relaciona más bien con el hecho que sería un producto de menor costo.

Finalmente destaca que cerca del $60 \%$ de las madres no comparte la bebida láctea que recibe en el consultorio, lo que indica una dilución intrafamiliar relativamente baja y un buen posicionamiento del producto para el

\section{TABLA 2}

\section{Distribución según cantidad diaria de leche consumida en el período pregestacional y de bebida Láctea Purita Mamá durante el embarazo y lactancia.}

\begin{tabular}{|c|c|c|c|c|}
\hline \multirow[t]{2}{*}{ Consumo de leche } & \multicolumn{2}{|c|}{ Embarazadas } & \multicolumn{2}{|c|}{ Nodrizas } \\
\hline & $\begin{array}{c}\text { Previo al } \\
\text { embarazo } \\
\%\end{array}$ & $\begin{array}{c}\text { Durante el } \\
\text { embarazo } \\
\%\end{array}$ & $\begin{array}{c}\text { Previo al } \\
\text { embarazo } \\
\%\end{array}$ & $\begin{array}{c}\text { Durante la } \\
\text { lactancia } \\
\%\end{array}$ \\
\hline No consumía leche & 35,1 & 17,3 & 29,9 & 17,4 \\
\hline 1 taza o menos $(<200 \mathrm{~mL})$ & 31,0 & 31,1 & 21,4 & 22,2 \\
\hline 1 a 2 tazas $(201-400 \mathrm{~mL})$ & 20,8 & 29,2 & 25,3 & 30,6 \\
\hline Más de 2 tazas (> $401 \mathrm{~mL})$ & 13,1 & 22,4 & 23,4 & 29,8 \\
\hline Total & 100,0 & 100,0 & 100,0 & 100,0 \\
\hline$\chi^{2} \mathrm{p}$ & \multicolumn{2}{|c|}{$48,27<0,001$} & \multicolumn{2}{|c|}{$68,23<0,001$} \\
\hline
\end{tabular}

\section{TABLA 3}

\section{Opinión de la madre sobre el motivo del MINSAL para entregar a bebida láctea Purita Mamá a embarazadas y nodrizas.}

\begin{tabular}{lccc} 
Motivos & $\begin{array}{c}\text { Embarazadas } \\
\mathbf{\%}\end{array}$ & $\begin{array}{c}\text { Nodrizas } \\
\mathbf{\%}\end{array}$ & $\begin{array}{c}\text { Total } \\
\mathbf{\%}\end{array}$ \\
\hline Es más barata & 3,5 & 3,0 & 3,2 \\
Mas nutritiva para la madre & 38,8 & 37,2 & 38,0 \\
Ventajas para el hijo & 47,5 & 50,2 & 48,9 \\
No sabe & 10,2 & 9,6 & 9,9 \\
Total & $\mathbf{1 0 0 , 0}$ & $\mathbf{1 0 0 , 0}$ & $\mathbf{1 0 0 , 0}$ \\
$\chi 2$ p & & 0,99 p NS &
\end{tabular}


grupo de beneficiarios al que esta destinado. Cerca de un tercio lo comparte con otros miembros del hogar, lo que también refleja indirectamente una buena aceptación del producto (tabla 4).

\section{DISCUSIÓN}

En base a toda la evidencia mundial existente, las enormes ventajas de un mayor aporte de DHA en el embarazo y lactancia están fuera de discusión (20-25). También está bien documentado el bajo consumo de pescado y de DHA en la población chilena $(19,26)$, lo que refleja la necesidad de aumentar la ingesta a través de diversas opciones: educación alimentaria, suplementos de DHA o uso de alimentos fortificados. El Programa Nacional de Alimentación Complementaria ofrece la gran oportunidad de llegar a la gran mayoría de la población más vulnerable del país y es la opción que adoptó el Ministerio a partir de mediados del 2008.

Los alimentos especialmente formulados tienen la ventaja de entregar un aporte nutricional adecuado a las necesidades específicas de un grupo determinado de población. Esa es una de las grandes ventajas de la bebida láctea Purita Mama, que tiene un aporte reducido de grasas, lactosa y sodio y que está fortificada con vitaminas, minerales y DHA de acuerdo a las recomendaciones nutricionales para la embarazada y nodriza.

Sin embargo, un enorme desafío de los programas que incorporan alimentos que no son de consumo habitual, es lograr un adecuado nivel de aceptabilidad y consumo. Diferentes países de la región han incorporado a sus programas mezclas proteicas (Incaparina, Bienestarina, Nutricereal, Mi Sopita) con diferente grado de éxito (27-29). La mayoría de los programas considera un consumo diario del producto y más de una ración diaria, lo que es muy difícil de lograr. A menudo la frecuencia de consumo es sólo algunos días a la semana, en menor volumen del programado y con un importante grado de dilución intrafamiliar $(30,31)$.

Las bebidas lácteas tienen la ventaja de ser un sustituto de la leche, alimento de excelente aceptabilidad e imagen, lo que puede favorecer su consumo. Esa fue la experiencia en Chile con el producto Purita Cereal para los preescolares y la bebida láctea "Años Dorados" del adulto mayor, que en general han sido muy bien aceptadas por la población beneficiaria (31).

Los resultados no han sido satisfactorios para la bebida láctea Purita Mama, ya que el producto ha generado algún grado de rechazo en la población, inicialmente por su sabor y olor a vainilla y por su dulzor. En la encuesta del 2009 el 81,4\% de las beneficiarias quería hacer cambios al producto, especialmente reducir el sabor a vainilla (38\%), el olor a vainilla y el grado de dulzor (32). Por otro lado con alguna frecuencia los profesionales de atención primaria reciben la queja que el producto tiene "olor a pescado", aunque ello no ha sido objetivado a través de paneles de expertos, lo que sugiere la posibilidad de que se generaron prejuicios infundados en relación al alimento (18).

Es destacable la posición del Ministerio de Salud de evaluar la aceptabilidad del producto y utilizar sus resultados para realizar los cambios necesarios, a un año de iniciada la distribución. Ello permitió desarrollar nuevos prototipos, que fueron analizados por paneles de expertos y posteriormente probados durante un mes en dos consultorios del sistema público de salud. Con frecuencia los Ministerios de Salud o de Protección Social mantienen los programas alimentarios por años, sin tener información básica sobre la percepción de los beneficiarios.

Esta segunda encuesta tuvo el propósito de evaluar si la apreciación y consumo de la población había mejorado. La encuesta mostró algunos resultados positivos,

\section{TABLA 4}

Distribución según el uso de la bebida láctea Purita Mamá por tipo de beneficiaria.

\section{Uso de Purita Mamá}

La consume la beneficiaria

Comparte con niños de la familia

Comparte con adultos de la familia

La regala

Total

$\chi^{2} \mathrm{p}$

Embar

66,3

10,7

15,5

7,5

100,0
Nodrizas

(\%)

53,0

14,3

22,5

10,2

100,0
Total

(\%)

59,7

12,5

19,0

8,8

100,0

$20,69 p<0,001$ 
como una buena adherencia al programa (el 90\% de los beneficiarios lo retira regularmente), buena opinión sobre sus características nutricionales y un nivel de consumo en embarazadas superior a la ingesta habitual de leche en el período pregestacional y cercano a la meta esperada. Aunque el consumo aumenta en la lactancia, no lo hace en la misma proporción en función de la mayor cantidad recibida. También es destacable que en el $60 \%$ de las familias la madre sea la única consumidora de Purita Mama y que menos del 10\% reconoce que regala la bebida láctea Purita Mamá.

Sin embargo, persisten aspectos negativos, que es necesario abordar para mejorar el impacto del programa. Cerca de $7 \%$ de las madres no retira el producto, porque no le gusta o le hace mal, lo que sugiere la necesidad de revisar nuevamente sus características organolépticas. Ello se refleja también en la apreciación organoléptica, con una nota "regular" asignada al producto y en el porcentaje de mujeres que no valora bien su sabor, olor o textura. Llama también la atención que un porcentaje de la población aún lo considere "dulce", aunque una ración de $200 \mathrm{~mL}$ aporta sólo 1,2 g de sacarosa. A pesar de estos hechos negativos, el consumo real en embarazadas esta muy cercano a lo programado.

Aún cuando la inmensa mayoría de las madres valora sus características nutricionales, cerca de un $10 \%$ desconoce el por qué el Ministerio está utilizando este producto. Por lo tanto, es importante que el personal de atención primaria refuerce la información a las madres sobre las bondades del producto.

Otro hecho que llama la atención es que menos del $25 \%$ de las madres preparare el producto según las normas establecidas, lo que sin duda puede afectar la opinión sobre la textura, sabor u olor del alimento. Estos resultados en parte podrían explicarse por problemas relacionados con la confiabilidad de la información obtenida, pero sugieren la necesidad de reforzar ese aspecto en los controles de salud y eventualmente incorporar un medidor para mejorar la preparación del producto.

Un problema adicional, es que de acuerdo a datos entregados por los profesionales de atención primaria, una proporción de las madres refiere haber suspendido la lactancia materna para recibir la leche Purita Fortificada, alimento de amplia aceptabilidad. Este hecho podría explicar en parte la reducción de la lactancia materna exclusiva a los 6 meses de edad del niño, de 49 a $45 \%$ en los últimos dos años. La Leche Purita Fortificada tiene una muy buena aceptabilidad, pero a pesar de ello el consumo real es similar al de Purita Mama, porque una proporción mayor de madres la comparte con el resto de la familia.

Es difícil determinar cuál debiera ser la respuesta más adecuada: ¿reformular nuevamente el producto?, ¿reforzar la capacitación al equipo de salud y entregar mayor información a la comunidad?, ¿volver a la leche fortificada? Las dos primeras estrategias son válidas y complementarias y habría que implementarlas, aunque es difícil generar un "nuevo" alimento, que tenga una amplia aceptación de toda la población. La leche, a pesar de su buena aceptabilidad, no tiene un consumo real mayor que este producto. Otra potencial ventaja de continuar con esta bebida láctea es que podría existir algún grado de auto focalización de las beneficiarias, ya que no incentivaría a las madres de mejor nivel socioeconómico a retirar el producto.

De acuerdo a los resultados del estudio se considera indispensable reforzar la educación nutricional sobre la calidad y ventajas nutricionales del producto, tanto en las beneficiarias como al equipo de salud, a través de talleres, material educativo, degustaciones, entre otras. También es fundamental reforzar la información sobre la forma de preparar el producto y eventualmente entregar medidores, considerando que menos del $30 \%$ de las madres lo diluye en el rango recomendado. Es importante también proponer alternativas de preparación y consumo de Purita Mamá, especialmente para aquellas madres que no la consumen en forma líquida. Considerando que aún un grupo de madres tiene algún rechazo por el sabor y olor del producto, habría que desarrollar y evaluar nuevos prototipos, de sabor neutro.

Eventualmente podrían considerarse diferentes alternativas de sabor, a elección por la madre, aunque ello complica la logística del programa. Se considera que es importante continuar evaluando la aceptabilidad y consumo del producto, para redefinir las estrategias a seguir y cumplir adecuadamente con los objetivos del programa. Todas estas actividades permiten responder a los desafíos que exige una política pública, y brindar respuesta a las demandas de la población usuaria de este histórico e importante programa del Ministerio de Salud.

\section{RESUMEN}

Antecedentes: el Ministerio de Salud incorporó el año 2008 en el Programa Nacional de Alimentación Complementaria una bebida láctea para embarazadas y mujeres en lactancia, fortificada con EPA y DHA. El producto presentó inicialmente algún grado de rechazo en la población, lo que motivó modificar sus características organolépticas. Objetivo: evaluar en una muestra nacional la aceptabilidad y consumo de la "Purita Mama" modificada, un año después de iniciar su distribución. Metodología: muestra aleatoria de 1.272 beneficiarias en 62 Consultorios y 21 Postas Rurales, de todo el país. Se aplicó una encuesta semi estructurada para evaluar 
retiro, aceptabilidad y consumo por las beneficiarias y otros miembros del hogar. Resultados: El 90,7\% de las madres retiraba regularmente el producto. Se observaron importantes problemas en la preparación de Purita Mamá, por baja dilución (22\%) o excesiva concentración $(50,8 \%)$. La apreciación global del producto presentó una mediada de 3 en escala de 1 a 5 (regular) en embarazadas y nodrizas, destacando opiniones negativas respecto al excesivo dulzor $(19,2 \%)$ y aroma fuerte $(49,9 \%)$. La mediana de consumo de las embarazadas fue $250 \mathrm{~mL} /$ día, cercano a lo programado y algo superior en las nodrizas, aunque reciben mayor cantidad. El 86\% de las madres valora positivamente las características nutricionales del producto para la madre o su hijo. Cerca del $60 \%$ de las madres no comparte la bebida láctea, lo que indica una dilución intrafamiliar relativamente baja. Conclusiones: no hubo un cambio importante en el nivel de aceptabilidad del producto y los niveles de consumo fueron relativamente adecuados. Es importante reforzar la información sobre la forma de preparar el producto, proponer diferentes alternativas de preparación y eventualmente considerar alternativas de sabor.

Palabras clave: embarazada; nodriza; programas alimentarios; bebida láctea; evaluación

Dirigir la correspondencia a:

Profesora

Angela Contreras M.

Departamento de Alimentos y Nutrición,

División de Políticas Públicas Saludables y

Promoción, Subsecretaria de Salud Pública,

Ministerio de Salud,

Mac Iver 451

Santiago-Chile.

Fono: 56-2-5740474.

E-mail acontreras@minsal.cl

Agradecimientos: Lo autores agradecen a los encargados de los programas alimentarios y nutrición de las SEREMIS de Salud y a los equipos de salud que participaron en la coordinación y ejecución de la encuesta nacional, por la valiosa y eficiente colaboración brindada.

\section{BIBLIOGRAFÍA}

1. Carlson SE. Docosahexaenoic acid supplementation in pregnancy and lactation. Am J Clin Nutr 2009; 89: 678S-84S.

2. Koletzko B, Lien E, Agostoni C, Böhles H, Campoy C, Cetin I, Decsi T, Dudenhausen JW, Dupont C, Forsyth S, Hoesli I, Holzgreve W, Lapillonne A, Putet G, Secher NJ, Symonds M, Szajewska H,
Willatts P, Uauy R. The roles of long-chain polyunsaturated fatty acids in pregnancy, lactation and infancy: review of current knowledge and consensus recommendations. J Perinat Med 2008; 36: 5-14.

3. Szajewska H, Horvath A, Koletzko B. Effect of n-3 long-chain polyunsaturated fatty acid supplementation of women with low-risk pregnancies on pregnancy outcomes and growth measures at birth: a meta-analysis of randomized controlled trials. Am J Clin Nutr 2006; 83: 1337-44.

4. Mendez MA, Torrent M, Julvez J, Ribas-Fitó N, Kogevinas M, Sunyer J. Maternal fish and other seafood intakes during pregnancy and child neurodevelopment at age 4 years. Public Health Nutr 2008; 25:1-9.

5. Geleijnse JM, Giltay EJ, Schouten EG, de Goede J, Oude Griep LM, Teitsma-Jansen AM, Katan MB, Kromhout D. Effect of low doses of n-3 fatty acids on cardiovascular diseases in 4,837 post-myocardial infarction patients: design and baseline characteristics of the Alpha Omega Trial. Am Heart J 2010; 159: 539-46.

6. Kelley DS, Siegel D, Fedor DM, Adkins Y, Mackey BE. DHA supplementation decreases Serum Creactive protein and other markers of inflammation in hypertriglyceridemic men. J Nutr 2009;139: 495-501.

7. Harris WS, Kris-Etherton PM, Harris KA. Intakes of long-chain omega-3 fatty acid associated with reduced risk for death from coronary heart disease in healthy adults. Curr Atheroscler Rep 2008; 10: 503-509.

8. Lukiw WJ, Bazan NG. Docosahexaenoic acid and the aging brain. J Nutr 2008;138: 25: 10-4.

9. Pomponi M, Di Gioia A, Bria P, Pomponi MF. Fatty aspirin: a new perspective in the prevention of dementia of Alzheimer's type?. Curr Alzheimer Res 2008;5 : 422-31.

10. Samieri C, Féart C, Letenneur L, Dartigues JF, Pérès K, Auriacombe S, Peuchant E, Delcourt C, Barberger-Gateau P. Low plasma eicosapentaenoic acid and depressive symptomatology are independent predictors of dementia risk. Am J Clin Nutr 2008; 88: 714-21.

11. Valenzuela R, Bascuñan K, Valenzuela A, Chamorro R. Acidos grasos omega-3, enfermedades psiquiátricas y neurodegenerativas: un nuevo enfoque preventivo y terapéutico. Rev Chil Nutr 2009; 36: 1120-8.

12. Welch AA, Shakya-Shrestha S, Lentjes MA, Wareham NJ, Khaw KT. Dietary intake and status of n-3 polyunsaturated fatty acids in a population of 
fish-eating and non-fish-eating meat-eaters, vegetarians, and vegans and the precursor-product ratio of $\alpha$-linolenic acid to long-chain n-3 polyunsaturated fatty acids: results from the EPIC-Norfolk cohort. Am J Clin Nutr 2010; 92: 1040-51.

13. Talahalli RR, Vallikannan B, Sambaiah K, Lokesh BR. Lower efficacy in the utilization of dietary ALA as compared to preformed EPA + DHA on long chain n-3 PUFA levels in rats. Lipids 2010; 45: 799-808.

14. Valenzuela A, Sanhueza J. Aceites de origen marino; su importancia en la nutrición y en la ciencia de alimentos. Rev Chil Nutr 2009; 36: 246-57.

15. Calder PC, Dangour AD, Diekman C, Eilander A, Koletzko B, Meijer GW, Mozaffarian D, Niinikoski H, Osendarp SJ, Pietinen P, Schuit J, Uauy R. Essential fats for future health. Proceedings of the 9th Unilever Nutrition Symposium, 26-27 May 2010. Eur J Clin Nutr 2010; 64 Suppl 4:S1-13.

16. Atalah E, Araya H, Rosselot G, Pinheiro AC, Araya $\mathrm{M}$, Vargas V. Consumo de pescado, mariscos, EPA y DHA en gestantes de la Región Metropolitana. Primer Congreso Chileno de Salud Pública, Determinantes de la Salud: Resumen 17, Santiago, Chile, 2007.

17. Franke C, Verwied-Jorky S, Campoy C, Trak-Fellermeier M, Decsi T, Dolz V, Koletzko B. Dietary intake of natural sources of docosahexaenoic acid and folate in pregnant women of three European cohorts. Ann Nutr Metab 2008; 53: 167-74.

18. Atalah E, Vera G, Rosselot G, Araya H, Andreu R, Alviña A, Araya M. Desarrollo, consumo y aceptabilidad de una Bebida Láctea con DHA para embarazadas y nodrizas. Rev Chil Nutr 2008; 35: 433-42.

19. Atalah E, Araya M, Rosselot G, Araya H, Vera G, Andreu R, Barba C, Rodríguez L. Efecto de una Bebida Láctea con DHA en la composición de ácidos grasos del glóbulo rojo, de la leche materna y en el recién nacido. Arch Latinoam Nutr 2009; 59: 271-6.

20. Dunstan JA, Mitoulas LR, Dixon G, Doherty DA, Hartmann PE, Simmer K, Prescott SL. The effects of fish oil supplementation in pregnancy on breast milk fatty acid composition over the course of lactation: a randomized controlled trial. Pediatr Res 2007; 62: 689-94.

21. Van Goor SA, Dijck-Brouwer DA, HaddersAlgra M, Doornbos B, Erwich JJ, Schaafsma A, Muskiet FA. Human milk arachidonic acid and docosahexaenoic acid contents increase following supplementation during pregnancy and lactation. Prostaglandins Leukot Essent Fatty Acids 2009;
80: 65-9.

22. Krauss-Etschmann S, Shadid R, Campoy C, Hoster E, Demmelmair H, Jiménez M, Gil A, Rivero M, Veszprémi B, Decsi T, Koletzko BV. Effects of fishoil and folate supplementation of pregnant women on maternal and fetal plasma concentrations of docosahexaenoic acid and eicosapentaenoic acid: a European randomized multicenter trial. Am J Clin Nutr. 2007; 85:1392-400.

23. Judge MP, Harel O, Lammi-Keefe CJ. A docosahexaenoic acid-functional food during pregnancy benefits infant visual acuity at four but not six months of age. Lipids 2007; 42:117-22.

24. Koletzko B, Cetin I, Brenna JT; Perinatal Lipid Intake Working Group. Dietary fat intakes for pregnant and lactating women. Br J Nutr 2007; 98: 873-7.

25. Van de Rest O, Geleijnse JM, Kok FJ, van Staveren WA, Dullemeijer C, Olderikkert MG, Beekman AT, de Groot CP. Effect of fish oil on cognitive performance in older subjects: a randomized, controlled trial. Neurology 2008;71: 430-8.

26. MINSAL Encuesta_Nacionalde_Salud ENS 20092010. http:/ www.redsalud.gov.cl/portal/ url / item 19ac1bf1ed8ee8922e04001011e013.pdf

27. Escalante-Izeta, E, Bonvecchio A, Théodore F; Nava F, Villanueva MA, Rivera-Dommarco, J. Facilitadores y barreras para el consumo del complemento alimenticio del Programa Oportunidades. Salud Pub México 2008; 50: 316-24.

28. De Caballero E, Atalah E. Evaluación de la aceptabilidad y consumo de un suplemento alimentario en la República de Panamá. Rev Chil Nutr 2003; 30: 133-140.

29. Martínez H, Campero L, Rodríguez G, Rivera, J. Aceptabilidad a suplementos nutricios en mujeres embarazadas o lactando y niños menores de cinco años. Salud Pub México 1999; 41: 163-9.

30. Pizarro T, Rodríguez L, Benavides X, Atalah E. Evaluación de la aceptabilidad y consumo de un nuevo producto del Programa Nacional de Alimentación Complementaria: Mi Sopita. Rev Chil Ped 2006; 77: 356-62.

31. Masi C, Atalah E. Análisis de la aceptabilidad, consumo y aporte nutricional del programa alimentario del adulto mayor. Rev Méd Chile 2008; 136: 415-22.

32. Ministerio de Salud. Encuesta Nacional de Aceptabilidad de la Bebida Láctea Purita Mama, Santiago, 2009 http://www.redsalud.gov.cl/portal/url/item/94 90e30c2b65eafbe04001011f017740.pdf 